TITLE:

\title{
Vibrational overtone dephasing in liquids under the influence of non- Gaussian noise
}

$\operatorname{AUTHOR}(S):$

Yamaguchi, T

\section{CITATION:}

Yamaguchi, T. Vibrational overtone dephasing in liquids under the influence of nonGaussian noise. JOURNAL OF CHEMICAL PHYSICS 2000, 112(19): 8530-8533

\section{ISSUE DATE:}

2000-05-15

URL:

http://hdl.handle.net/2433/49896

\section{RIGHT:}

Copyright 2000 American Institute of Physics. This article may be downloaded for personal use only. Any other use requires prior permission of the author and the American Institute of Physics. 


\title{
Vibrational overtone dephasing in liquids under the influence of non-Gaussian noise
}

\author{
T. Yamaguchi ${ }^{\mathrm{a})}$ \\ Department of Chemistry, Graduate School of Science, Kyoto University, Kyoto 606-8502, Japan
}

(Received 14 January 2000; accepted 22 February 2000)

\begin{abstract}
We have calculated the vibrational overtone dephasing rates of an oscillator that is subject to uncorrelated, impulsive, and strong collisions. We have assumed the Poisson statistics for binary collisions, and not truncated the cumulant expansion at the second order. The decay of the response function is always exponential, and the dephasing rate shows subquadratic dependence on the vibrational quantum number $(\nu)$. The dephasing rate asymptotically converges to the collision frequency at the large- $\nu$ or strong collision limits. We have succeeded in reproducing the experimental results on chloroform [K. Tominaga and K. Yoshihara, J. Phys. Chem. A 102, 4222 (1998)] and those on methyl iodide [A. B. Myers and F. Markel, Chem. Phys. 149, 21 (1990)] by adjusting the collision frequency and the strength of the collision. (C) 2000 American Institute of Physics. [S0021-9606(00)50519-4]
\end{abstract}

\section{INTRODUCTION}

In the stochastic theory of the optical or the magnetic resonance spectra in liquids, the chromophores (spin, vibration, and so on) are often treated as the quantum systems whose transition frequencies fluctuate stochastically due to the interaction with surroundings. ${ }^{1,2}$ The fluctuation of the transition energy is usually assumed to be a Gaussian process, and the cumulant expansion is truncated at the second order, which decreases the numerical efforts very much. This approximation is guaranteed by the central limiting theorem, which states that a stochastic process becomes the Gaussian process when it is the accumulation of many uncorrelated small elements. ${ }^{3}$ The Brownian oscillator model for nonlinear optical processes in liquids has been developed, which takes advantage of the fact that the Gaussian process is expressed by an infinite number of harmonic oscillators. ${ }^{4}$

However, there are some cases where the chromophore is coupled strongly to a small number of solvent molecules. In such cases, there is no reason to guarantee that the fluctuation is a Gaussian process. One example is the hydrogen bonding system. Another example is the vibrational dephasing due to the repulsive interaction, which is the theme of this work. A molecule in liquids always experiences repulsive collisions that perturb the phase of the intramolecular vibration. This perturbation is the origin of the vibrational dephasing due to the repulsive interaction. The vibrational spectra of many molecules have been explained by the vibrational dephasing due to the repulsive interaction, which is sometimes treated by the isolated binary collision (IBC) model. The distribution of the number of uncorrelated collisions shows a Poisson distribution, not a Gaussian one. For instance, Frankland and Maroncelli showed that the distribution of the instantaneous transition frequency deviated to a large degree from the Gaussian distribution in their molecular dynamics simulation..$^{5}$ Therefore, we need to calculate the

${ }^{a}$ Electronic mail: tyamaguchi@kuchem.kyoto-u.ac.jp vibrational spectra in this case not by truncating the cumulant expansion at the second order. We actually perform such a calculation in this work, and show that the non-Gaussian effect becomes large, especially for overtone dephasing. Although there are many studies on the Poisson statistics itself, $^{3}$ as far as we know, this is the first study on its application to the vibrational dephasing in liquids.

The quantum number $(\nu)$ dependence of the vibrational dephasing rates has long been an interesting issue in connection with the mechanism of the vibrational dephasing. The vibrational spectra $[I(\omega)]$ is the Fourier transform of $I(t)$ (hereafter called the "response function") as follows:

$$
I(\omega)=\operatorname{Re} \int_{0}^{\infty} d t e^{i \omega t} I(t)
$$

where the response function $[I(t)]$ is defined as follows:

$$
I(t)=\left\langle\exp \left[-i \omega^{0} t-i \int_{0}^{t} d t^{\prime} \delta \omega\left(t^{\prime}\right)\right]\right\rangle .
$$

In Eq. (2), the angular bracket means the statistical average, and $\omega^{0}$ and $\delta \omega\left(t^{\prime}\right)$ stand for the transition frequency in the gas phase and the instantaneous transition frequency shift at $t=t^{\prime}$ due to the solvents, respectively. The instantaneous frequency shift $[\delta \omega(t)]$ is proportional to the instantaneous random force along the vibrational normal mode from the solvent in the theory developed by Oxtoby. ${ }^{6}$ In a standard procedure, ${ }^{2}$ we assume that $\delta \omega(t)$ is a Gaussian process and symmetric with respect to the time translation and inversion. Under these assumptions, the cumulant expansion of Eq. (2) can be truncated at the second order to yield the following equation: 


$$
\begin{aligned}
I(t)= & \exp \left(-i\left(\omega^{0}+\langle\delta \omega\rangle\right) t\right. \\
& \left.-\int_{0}^{t} d t^{\prime}\left[\left(t-t^{\prime}\right)\left(\left\langle\delta \omega(0) \delta \omega\left(t^{\prime}\right)\right\rangle-\langle\delta \omega\rangle^{2}\right)\right]\right) .
\end{aligned}
$$

In the fast modulation case, where the relaxation of $\langle\delta \omega(0) \delta \omega(t)\rangle$ is very fast (compared with the vibrational dephasing), Eq. (3) reduces to the following equation:

$$
\begin{aligned}
I(t)= & \exp \left(-i\left(\omega^{0}+\langle\delta \omega\rangle\right) t-t\right. \\
& \left.\times \int_{0}^{t} d t^{\prime}\left[\left\langle\delta \omega(0) \delta \omega\left(t^{\prime}\right)\right\rangle-\langle\delta \omega\rangle^{2}\right]\right),
\end{aligned}
$$

and the time profile of the response function is exponential. Even if the relaxation of $\delta \omega(t)$ is not so fast, the asymptotic behavior of the response function is always exponential, as long as the correlation time of $\delta \omega(t)$ is finite.

In the opposite limit, called the "slow modulation limit,' Eq. (3) reduces to the following equation:

$$
I(t)=\exp \left(-i\left(\omega^{0}+\langle\delta \omega\rangle\right) t-\frac{t^{2}}{2}\left(\left\langle\delta \omega^{2}\right\rangle-\langle\delta \omega\rangle^{2}\right)\right),
$$

and the bandshape of the spectrum is a Gaussian function.

The fluctuation of the transition frequency of the $\nu$-phonon transition $\left(\delta \omega_{\nu}\right)$ is $\nu$ times as large as that of the fundamental vibrational frequency $\left(\delta \omega_{1}\right)$ in the case of the harmonic oscillator as follows:

$$
\delta \omega_{\nu}=\nu \delta \omega_{1} .
$$

From Eqs. (4)-(6), the bandwidths of the overtone spectra are proportional to $\nu^{2}$ in the fast modulation case, whereas they are proportional to $\nu$ in the slow modulation limit. Therefore, the quantum number dependence of the vibrational bandwidths can be a measure to distinguish the fast and the slow modulation limits of the vibrational dephasing.

The correlation time of $\delta \omega(t)$ depends on its molecular origin. For example, the fast modulation limit is expected for the repulsive collisions, whereas the attractive interaction is considered to belong to the slow modulation limit. ${ }^{7,8}$ The overtone spectra ( $\nu=2$ transition) for some molecules have been measured by IR or nonresonant Raman spectroscopy in the frequency domain, and it is found that the ratio of the bandwidth of the overtone and fundamental is smaller than 4 for many molecules. ${ }^{9-11}$ The progressions more than $\nu=10$ were measured for iodine ${ }^{12}$ and methyl iodide ${ }^{13}$ by the resonance Raman spectroscopy. The bandwidths appeared proportional to $\nu^{2}$ for small $\nu$, and linear to $\nu$ for large $\nu$. Therefore they explained the $\nu$ dependence of the bandwidth by the transition from the fast to the slow modulation limits. However, their correlation times (330 fs for iodine, and 500 fs for methyl iodide) were claimed to be somewhat large for the collisional interaction. Recently, Tominaga and Yoshihara performed the time domain experiments on the overtone dephasing of the $\mathrm{C}-\mathrm{H}$ stretching mode of chloroform, and found that the quantum number dependence of the dephasing rates is smaller than $\nu^{2}$, although the time profile of the response function is exponential for overtones. ${ }^{14-16}$ Their results contradict to the standard theory [Eqs. (3)-(6)], since the exponential profile of the response function means the fast modulation limit, where $\nu^{2}$ dependence of the dephasing rate is expected. Considering that the $\nu^{2}$ dependence holds in the case of iodine, Tominaga and Yoshihara suggested that the polyatomic nature play a key role in the vibrational dephasing. ${ }^{16}$ Gayathri et al. ${ }^{17}$ claimed that the subquadratic $\nu$ dependence is explained by the bimodality of the time correlation function of $\delta \omega(t)$, which is calculated by the modecoupling theory or the molecular dynamic simulation. ${ }^{17}$ The slow component of $\langle\delta \omega(0) \delta \omega(t)\rangle$ can reproduce the subquadratic $\nu$ dependence of the average dephasing rate or the total bandwidth, which is actually the transition between the fast and the slow modulation limits. However, the time constant of the asymptotic exponential decay of the response function should always be proportional to $\nu^{2}$ in the standard theory, which contradicts with the experimental results of Tominaga and Yoshihara. ${ }^{16}$ In this work, we shall show that the subquadratic $\nu$ dependence is consistent with the exponential decay of the response function when $\delta \omega(t)$ is not the Gaussian process.

\section{MODEL AND CALCULATION}

In our model, we suppose that an oscillator in solution experiences a number of impulsive and uncorrelated binary collisions to yield $\delta \omega_{1}(t)$ as follows:

$$
\delta \omega_{1}(t)=\sum_{j} \delta \phi_{1, j} \delta\left(t-t_{j}\right),
$$

where $t_{j}$ and $\delta \phi_{1, j}$ stand for the time and the phase shift of the $j$ th collision, respectively. The probability distribution of $\delta \phi_{1, j}\left[P_{\phi}\left(\delta \phi_{1}\right)\right.$, independent of $\left.j\right]$ is assumed to be exponential,

$$
P_{\phi}(\delta \phi)=\left\{\begin{array}{lc}
e^{-\delta \phi / \delta \phi^{0}} / \delta \phi^{0} & (\delta \phi>0) \\
0 & (\delta \phi<0)
\end{array} .\right.
$$

The value of $\delta \phi^{0}$ is the average phase shift per collision. No correlation is considered between $\delta \phi_{1, j}$ of different collisions. The distribution of Eq. (8) stems from the idea that the phase shift due to the collision will be proportional to the relative kinetic energy of the collision pair. The exponential distribution of the instantaneous frequency is observed in the molecular dynamics simulation of Frankland and Maroncelli. ${ }^{5}$ Equation (6) is assumed for the fluctuation of the transition frequency of overtones. Since there is no correlation between collisions, the distribution of the number of collisions between $[0, t]$ [denoted as $P_{c}(n, t)$, where $n$ stands for the number of collisions] should obey the Poisson distribution as

$$
P_{c}(n, t)=\frac{1}{n !}\left(\frac{t}{\tau_{c}}\right)^{n} \exp \left(-\frac{t}{\tau_{c}}\right) .
$$

In Eq. (9), $\tau_{c}^{-1}$ stands for the collision frequency.

In this model, the vibrational dephasing in the Gaussian approximation is easily obtained. At first, the time correlation function of $\delta \omega_{\nu}(t)$ is expressed as follows: 


$$
\left\langle\delta \omega_{\nu}(0) \delta \omega_{\nu}(t)\right\rangle=\nu^{2}\left(\delta \phi^{0}\right)^{2}\left[2 \tau_{c} \delta(t)+1\right] / \tau_{c}^{2} .
$$

Therefore, the response function for the $\nu$ th overtone $\left[I_{\nu}(t)\right]$ is obtained by substituting Eq. (10) into Eq. (3),

$$
I_{\nu}(t)=\exp \left[-i\left(\omega_{\nu}^{0}+\Delta \omega_{\nu}\right) t-t / T_{2, \nu}\right] .
$$

The value of $\omega_{\nu}^{0}$ stands for the transition frequency of the $\nu$ th overtone in the gas phase. The decay of the response function is always exponential (fast modulation limit), and the shift $\left(\Delta \omega_{\nu}\right)$ and the dephasing time $\left(T_{2, \nu}\right)$ are given in the following equations:

$$
\begin{aligned}
& \Delta \omega_{\nu}=\nu \delta \phi^{0} / \tau_{c}, \\
& T_{2, \nu}^{-1}=\nu^{2}\left(\delta \phi^{0}\right)^{2} / \tau_{c} .
\end{aligned}
$$

As is expected, the vibrational dephasing is in the fast modulation limit and the dephasing rate is proportional to $\nu^{2}$.

In the following, we calculate the vibrational dephasing from Eq. (2) without employing the cumulant expansion. At first, the rhs of Eq. (2) is classified by the number of collisions between $[0, t]$,

$I_{\nu}(t)=e^{-i \omega_{\nu}^{0} t} \sum_{k=0}^{\infty} P_{c}(k, t)\left[\int_{0}^{\infty} d(\delta \phi) P_{\phi}(\delta \phi) e^{-i \nu \delta \phi}\right]^{k}$.

The inner part of $[\cdots]$ is calculated by using Eq. (8) to yield the following expression:

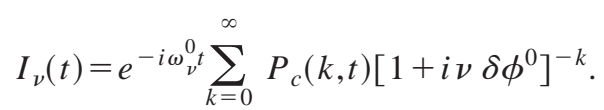

By substituting Eq. (9) into Eq. (15), $I_{\nu}(t)$ is obtained as follows:

$$
\begin{aligned}
e^{i \omega_{\nu}^{0} t} I_{\nu}(t) & =\sum_{k=0}^{\infty} \frac{1}{k !}\left\{\frac{t}{\tau_{c}\left(1+i \nu \delta \phi^{0}\right)}\right\}^{k} e^{-t / \tau_{c}} \\
& =\exp \left[-\frac{t}{\tau_{c}}\left\{1-\left(1+i \nu \delta \phi^{0}\right)^{-1}\right\}\right] \\
& =\exp \left[-i \Delta \omega_{\nu}-t / T_{2, \nu}\right] .
\end{aligned}
$$

The time profile of the response function is exponential, as is the case of Gaussian approximation. The shift $\left(\Delta \omega_{\nu}\right)$ and the dephasing time $\left(T_{2, \nu}\right)$ are given by the following expression:

$$
\begin{aligned}
\Delta \omega_{\nu} & =\frac{\nu \delta \phi^{0}}{\tau_{c}\left[1+\nu^{2}\left(\delta \phi^{0}\right)^{2}\right]}, \\
T_{2, \nu}^{-1} & =\frac{\nu^{2}\left(\delta \phi^{0}\right)^{2}}{\tau_{c}\left[1+\nu^{2}\left(\delta \phi^{0}\right)^{2}\right]} .
\end{aligned}
$$

The results of non-Gaussian process [Eqs. (17) and (18)] agrees with those of Gaussian approximation [Eqs. (12) and (13)] in the case of small $\delta \phi^{0}$ (weak collision) limit. It is quite reasonable, since the cumulant expansion is the perturbation theory with respect to $\delta \omega(t)$. In this model, on the other hand, the $\nu$ dependence of the dephasing rate is subquadratic in general, and $T_{2, \nu}$ reaches $\tau_{c}$ in the limits of large $\nu$ or large $\delta \phi^{0}$ (strong collision). In this limit, the collision between the solute and the solvent is the rate-determining step for the vibrational dephasing.
TABLE I. The dephasing times of the overtones of the $\mathrm{C}-\mathrm{H}$ stretching mode of chloroform $\left(\mathrm{CHCl}_{3}\right)$ and its deuterated version $\left(\mathrm{CDCl}_{3}\right)$. The calculation is based on Eq. (18) and compared with the experiment in Ref. 16. The parameters used in the calculation are $\tau_{c}=260 \mathrm{fs}$ (in common), $\left(\delta \phi^{0}\right)^{2}=0.33$ for the $\mathrm{C}-\mathrm{H}$ mode, and $\left(\delta \phi^{0}\right)^{2}=0.14$ for the $\mathrm{C}-\mathrm{D}$ mode. The dephasing time is given in units of ps.

\begin{tabular}{cccccc}
\hline \hline & \multicolumn{2}{c}{$\mathrm{CHCl}_{3}$} & & \multicolumn{2}{c}{$\mathrm{CDCl}_{3}$} \\
\cline { 2 - 3 } \cline { 5 - 5 } & Theory & Experiment & & Theory & Experiment \\
\hline$\nu=1$ & 1.05 & 1.06 & & 2.12 & 2.10 \\
$\nu=2$ & 0.46 & 0.42 & & 0.72 & 0.94 \\
$\nu=3$ & 0.35 & 0.30 & & 0.47 & 0.46 \\
\hline \hline
\end{tabular}

\section{RESULTS AND DISCUSSIONS}

Although our model can reproduce the subquadratic $\nu$ dependence of the dephasing rate and the exponential decay of the response function at the same time, this model is too simple to claim a quantitative agreement with the experiment. First, the finite duration of the repulsive collision is not included. Therefore, the transition between the fast and the slow modulation limits is not reproduced in our model. Second, the correlation between collisions, which is obvious in the case of self-diffusion as the deviation from the Enskog theory, ${ }^{18,19}$ is neglected. Third, dephasing mechanisms other than repulsive collisions are not considered, for example, attractive interaction, rotation-vibration coupling, resonant and nonresonant energy transfer, or intramolecular vibrational redistribution. However, surprisingly enough, our model can reproduce some experiments well by adjusting parameters.

First, we will apply this model to the $\mathrm{C}-\mathrm{H}$ stretching mode of chloroform and the $\mathrm{C}-\mathrm{D}$ stretching mode of chloroform- $d$ by Tominaga and Yoshihara. ${ }^{16}$ They measured the response function up to the $\nu=3$ transition in the time domain experiment, and found that the $\nu$ dependence of the dephasing rate is subquadratic (see Table I) and the decay of the response function is always exponential. We fitted their result by Eq. (18), and the best fit parameters are $\tau_{c}$ $=260 \mathrm{fs}$ (in common), $\left(\delta \phi^{0}\right)^{2}=0.33$ for the $\mathrm{C}-\mathrm{H}$ mode, and $\left(\delta \phi^{0}\right)^{2}=0.14$ for the $\mathrm{C}-\mathrm{D}$ mode. The theoretical values are compared with the experimental ones in Table I. The agreement is fairly good. However, there are at least two troubles in this calculation. The first one is that the collision time $\left(\tau_{c}\right)$ appears too large. For example, Schroeder et al. estimated the Enskog collision time of chloroform at $30^{\circ} \mathrm{C}$ and $30 \mathrm{bar}$ to be $110 \mathrm{fs} .^{20}$ The disagreement with the Enskog collision frequency may be explained by the steric factor. ${ }^{21}$ The second trouble in our model is the inability to reproduce the two-dimensional experiment by Tominaga and Yoshihara. ${ }^{15}$ The correlation between the two time intervals should not exist in our model, whereas it exists in the experiment. It seems to us that the correlation between collisions is essential to reproduce the two-dimensional experiment.

The second example is the $\mathrm{C}-\mathrm{I}$ stretching mode of methyl iodide in hexane measured by Myers and Markel. ${ }^{13}$ They measured the bandwidths up to $\nu=15$ by the ultraviolet resonance Raman spectroscopy. The bandwidths are quadratic with respect to the quantum number at small $\nu$, and the 


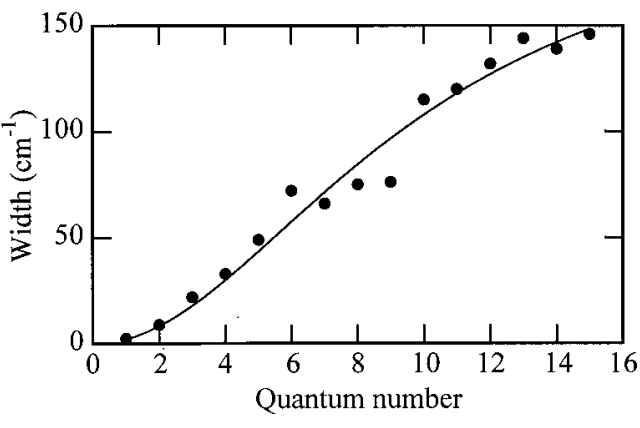

FIG. 1. The bandwidths (full width at half-maximum) of the overtones of the C-I stretching mode of methyl iodide in hexane. Experimental results (filled circles, Ref. 13) are compared with the theory [solid curves, Eq. (18)]. In the calculation, the values of $\tau_{c}$ and $\left(\delta \phi^{0}\right)^{2}$ (see the text) are assumed to be $50 \mathrm{fs}$ and 0.010 , respectively.

linear $\nu$ dependence is observed at large $\nu$ (Fig. 1). They could reproduce the bandwidths only if they assumed a very long correlation time (about $500 \mathrm{fs}$ ) for the repulsive interaction. The simulation by our model [Eq. (18)] is shown in Fig. 1. In the calculation, the values of $\tau_{c}$ and $\left(\delta \phi^{0}\right)^{2}$ (see the text) are assumed to be 50 fs and 0.010 , respectively. We consider it qualitatively reasonable that the collision frequency of methyl iodide in hexane is larger than that of chloroform in neat chloroform, partly because the iodine atom and the methyl group are much larger than the hydrogen atom. Since the $\nu$ dependence of the bandwidths appears almost linear at larger $\nu$ also in our model, it is difficult to distinguish experimentally the transition to the slow modulation limit in the standard theory and the collision limited saturation in our model from the bandwidths alone. We consider it desirable to determine the functional form of the response function in detail in order to clarify the mechanism of the vibrational dephasing. The overtone bandwidths of iodine were also measured by Battaglia and Madden. ${ }^{12}$ The widths are proportional to $\nu^{2}$ for small $\nu$, and the transition to the linear $\nu$ dependence is observed at large $\nu$. Although they interpreted their experiment as the transition to the slow modulation limit by assuming a somewhat large correlation time of $\delta \omega(t)$ (330 fs), we suspect there can be another explanation for their experiment. In our model, the difference between the quadratic $\nu$ dependence of iodine and the subquadratic $\nu$ dependence of many polyatomic molecules can be explained, not by the fact that the iodine is a diatomic molecule, but by the fact that the vibrational dephasing is much slower than the collision frequency in the case of iodine.

\section{SUMMARY}

In this work, we calculated the vibrational dephasing of a molecule under the influence of uncorrelated impulsive binary collisions without truncating the cumulant expansion at second order. We showed that the subquadratic quantum number dependence of the overtone dephasing rates and the exponential decay of the response function are not contradictory in the case of non-Gaussian noise. We could reproduce reported overtone dephasing rates of chloroform and methyl iodide fairly well by parameter optimization, and it can be safely said that the non-Gaussian effect can be important in the case of ultrafast vibrational dephasing due to collisional interaction. The subquadratic quantum number dependence of the overtone bandwidths of many molecules may suggest, we suspect, that the short time (high-frequency) behavior of the random force felt by a solute molecule is described better by the isolated binary collision model rather than by the Brownian oscillator model that assumes Gaussian noise.

\section{ACKNOWLEDGMENTS}

The author owes very much to Dr. Y. Kimura (Kyoto University) for discussions and reviewing the manuscript before submission. Professor Y. Tanimura (Institute for Molecular Science) and Professor N. Hirota (Kyoto University) should also be acknowledged for reading the manuscript. T. Y. is supported by the research fellowships from the Japan Society for the Promotion of Science (JSPS) for Young Scientists.

${ }^{1}$ R. Kubo, in Fluctuation, Relaxation, and Resonance in Magnetic Systems, edited by D. ter Harr (Plenum, New York, 1962).

${ }^{2}$ W. G. Rothschild, Dynamics of Molecular Liquids (Wiley, New York, 1984).

${ }^{3}$ See e.g., A. Papoulis, Probability, Random Variables, and Stochastic Processes (McGraw-Hill, New York, 1984).

${ }^{4}$ S. Mukamel, Principles of Nonlinear Optical Spectroscopy (Oxford University Press, New York, 1995).

${ }^{5}$ S. J. V. Frankland and M. Maroncelli, J. Chem. Phys. 110, 1687 (1999).

${ }^{6}$ D. W. Oxtoby, J. Chem. Phys. 70, 2605 (1979).

${ }^{7}$ K. S. Schweizer and D. Chandler, J. Chem. Phys. 76, 2296 (1982).

${ }^{8}$ We recently showed by a molecular dynamics simulation that the estimate of the correlation time by Schweizer and Chandler ${ }^{7}$ is an overestimate for the attractive interaction [T. Yamaguchi, Y. Kimura, and N. Hirota, J. Chem. Phys. 111, 4169 (1999)].

${ }^{9}$ C. Brodbeck, I. Rossi, Nguyen van Thanh, and A. Rouff, Mol. Phys. 32, 71 (1976).

${ }^{10}$ R. Arndt and J. Yarwood, Chem. Phys. Lett. 45, 155 (1977).

${ }^{11}$ J. Soussen-Jacob, C. Breuillard, J. Bessiere, J. Tsakiris, and J. VincentGeisse, Mol. Phys. 46, 545 (1982).

${ }^{12}$ M. R. Battaglia and P. A. Madden, Mol. Phys. 36, 1601 (1978).

${ }^{13}$ A. B. Myers and F. Markel, Chem. Phys. 149, 21 (1990).

${ }^{14}$ K. Tominaga and K. Yoshihara, Phys. Rev. Lett. 76, 987 (1996).

${ }^{15}$ K. Tominaga and K. Yoshihara, Phys. Rev. A 55, 831 (1997).

${ }^{16}$ K. Tominaga and K. Yoshihara, J. Phys. Chem. A 102, 4222 (1998).

${ }^{17}$ (a) N. Gayathri, S. Bhattacharyya, and B. Bagchi, J. Chem. Phys. 107, 10381 (1997); (b) N. Gayathri and B. Bagchi, ibid. 110, 539 (1999).

${ }^{18}$ B. J. Alder, D. M. Gass, and T. E. Wainwright, J. Chem. Phys. 53, 3813 (1970).

${ }^{19}$ T. Yamaguchi, Y. Kimura, and N. Hirota, Mol. Phys. 94, 527 (1998).

${ }^{20}$ J. Schroeder, V. H. Schiemann, and J. Jonas, Mol. Phys. 34, 1501 (1977).

${ }^{21}$ Looking at the time profile of the instantaneous frequency shift in the molecular dynamics simulation for the $\mathrm{C}-\mathrm{D}$ stretching mode of cyclohexane $d_{11},{ }^{5}$ it seems to us that the value of $\tau_{c}$ in our calculation is not so bad. 